\title{
Enhancing the sensitivity of a standard plasmonic MIM square ring resonator by incorporating nanodots in the cavity
}

\author{
N.L. Kazanskiy, ${ }^{1,2}$ M.A. Butt ${ }^{1}$ \\ ${ }^{1}$ Department of Technical Cybernetics, Samara National Research University, 34 Moskovkoye Shosse, \\ Samara 443086, Russia \\ ${ }^{2}$ Institute of RAS-Branch of the FSRC "Crystallography and Photonics" RAS, 151 Molodogvardeiskaya, \\ Samara 443001, Russia
}

Received October 06, 2019; accepted March 27, 2020; published March 31, 2020

\begin{abstract}
We studied the metal-insulator-metal square ring resonator design incorporated with nano-dots that serve to squeeze the surface plasmon wave in the cavity of the ring. The E-field enhances at the boundaries of the nano-dots provoking a strong interaction of light with the surrounding medium. As a result, the sensitivity of the resonator is highly enhanced compared to a standard ring resonator design. The best sensitivity of $907 \mathrm{~nm} / \mathrm{RIU}$ is obtained by placing seven nano-dots of radius $4 \mathrm{~nm}$ in all four sides of the ring with a period $\Lambda=3 \mathrm{r}$. The proposed design will find applications in biomedical science as highly refractive index sensors.
\end{abstract}

Surface plasmon polaritons (SPPs) are a kind of transverse EM-waves that can propagate along the boundary between metal and dielectric materials [1, 2], and can confine light in the sub-wavelength domain, overcoming the diffraction limit of light [3]. That is the reason why they are extensively used in highly integrated optical devices, such as optical switches [4], filters [5], sensors [6-8], nanowires [9], manipulation of light in MIM waveguides (WGs) [10], etc. In particular, the Metal-Insulator-Metal (MIM) plasmonic WG based on SPPs have been scrutinized in several applications such as plasmonic nano-sensors [11], plasmonic refractive index sensor [12], gear-shaped nano-cavities [13], plasmonic wavelength splitter [14]. Moreover, MIM WGs offer deep sub-wavelength confinement and low bend loss.

Optical sensors based on SPPs are extensively studied for the detection of refractive index (RI) changes of the materials due to their local field improvement and comparatively high sensitivity to the surrounding medium. Recently, several RI sensors having prominent designs have been proposed such as a plasmonic RI nanosensor based on MIM WG coupled, double rectangular cavities [15], asymmetric plasmonic WG comprised of two silver baffles and coupled ring resonators, RI sensor based on elliptical split nanorings [16], RI sensor based on Fano resonance in MIM WGcoupled trapezoid cavity [17]. Nevertheless, at the moment, these plasmonic sensors demonstrate low sensitivity, which is a big challenge to researchers. Biosensors are regularly used in numerous fields such as pharmaceutical industry, healthcare, biotechnology, food and environment monitoring to detect the interaction, presence and concentration of molecules [18].

In this work, we proposed a square ring resonator (SRR) incorporated with silver Nano-dots (NDs) sidecoupled by a MIM bus WG. The transmission spectrum and E-field distributions are simulated utilizing COMSOL 5.1 software employing a $2 \mathrm{D}$-finite element method (2D$F E M)$. The EM-wave frequency domain $(e m w)$ is selected as physics interface and modal analysis is added to the study. In COMSOL simulations, the subdomains of the device design are divided into triangular mesh elements with an extra-fine mesh grid size for the air medium and WG geometries. This produces precise simulation results within the available computational resources. When solving EM wave problems, it is beneficial to model a domain with open boundaries, that is such boundaries of the computational domain through which an EM wave can travel without reflection. To approximate an open geometry, scattering boundary conditions (SBC) are used at the outer edges of the FEM simulation window.

The metal and insulator materials used for the design are silver (Ag) and air, respectively. The frequencydependent permittivity of the $\mathrm{Ag}$ can be written by the Lorentz-Drude model [19]:

$$
\varepsilon_{m}(\omega)=\varepsilon_{\infty}-\frac{\omega_{p}^{2}}{\omega(\omega+i \gamma)} .
$$

The fundamental TM-mode of the MIM bus WG is excited by a plane wave. The width of the bus WG, side length of the ring, cavity width, gap between bus and ring are denoted by $w, L, c$ and $g$, respectively. The ring WG is connected to the output port which transmits the filtered wavelength depending on the resonance conditions. The resonance wavelength $\left(\lambda_{\text {res }}\right)$ of the ring resonator can be calculated by using the following expression:

$$
\lambda_{\text {res }}=\frac{4 L_{e f f} \operatorname{Re}\left(n_{e f f}\right)}{N},
$$

where $L_{\text {eff }}$ is the effective side length of the ring which is equal to the average of the inner and outer ring lengths of the square $(L+(L-c) / 2)$ and $n_{\text {eff }}$ denotes the effective RI in 
the square ring resonator. $N$ shows to the mode number which is an integer. It is visible from the equation that $\lambda_{\text {res }}$ has a direct relationship with RI of the medium and the side length of the resonator. The transmission spectrum is obtained by surface integration of the output port. There are seven NDs placed on each side of the cavity as shown in Fig. 1a. The NDs are placed in a cavity with a period $\Lambda$ of $3 \mathrm{r}$, where $r$ is the radius of the NDs. As mentioned earlier, the device design is divided into extra-fine triangular mesh elements as shown in Fig. 1b. The structural parameters of the sensor are presented in Table 1. Figure 1c shows the E-field distribution of the light in the standard ring design at corresponding $\lambda_{\text {res }}=$ $712 \mathrm{~nm}$. An array of NDs $(r=2 \mathrm{~nm})$ is placed in the cavity, which intensifies the resonating SPPs as shown in Fig. 1d. The inset shows the enhancement of SPPs at the boundaries of the NDs, which provokes strong interaction of light with the ambient medium and hence enhances the sensitivity of the sensor.

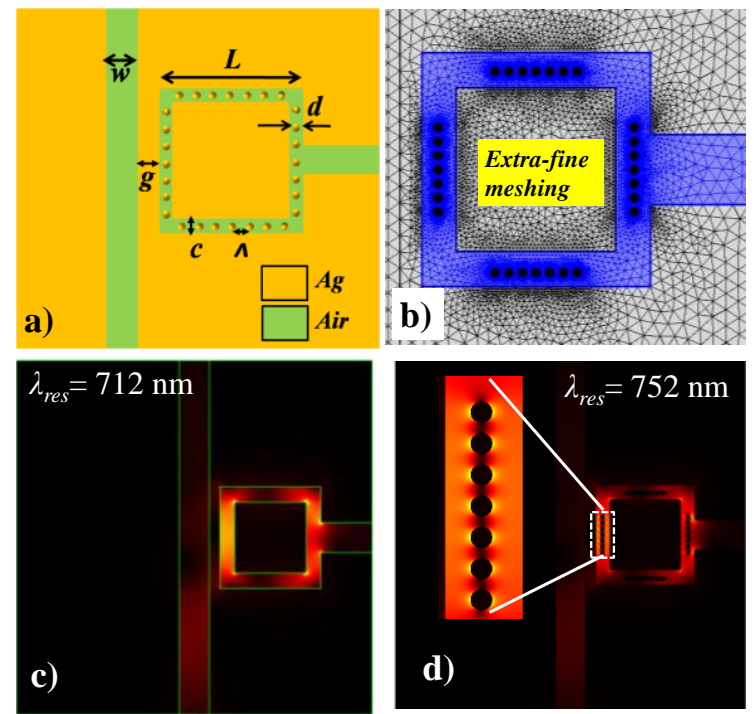

Fig. 1. a) Scheme of a proposed MIM SRR design with NDs incorporated in the cavity, b) Device design divided into extra-fine triangular mesh elements, c) E-field distribution in the standard SRR at $\lambda_{\text {res }}$, d) E-field distribution in the NDs incorporated SRR design at $\lambda_{\text {res }}$.

Table 1. Structural parameters of the sensor

\begin{tabular}{|c|c|c|c|c|}
\hline$W[\mathrm{~nm}]$ & $L[\mathrm{~nm}]$ & $g[\mathrm{~nm}]$ & $c[\mathrm{~nm}]$ & $r[\mathrm{~nm}]$ \\
\hline 30 & 100 & 10 & 15 & $2,2.5,3,3.5,4$ \\
\hline
\end{tabular}

We have discussed three significant factors such as sensitivity $(S=\Delta \lambda / \Delta n)$, the figure of merits $(F O M=S / F W H M)$ and the quality factor $(Q$ factor $=\lambda_{\text {res }}(F W H M)$, where $F W H M$ is the full width at half maximum, which is used to measure the sensing performance. $S$ is one of the most imperative figures of merits which should be considered. For sensing applications, FOM is usually applied to further approximate the sensing performance of the sensor which is wavelength dependent, while comparison between the needs should be taken into consideration. Integrated resonators with a high $Q$-factor are required for a wide range of applications such as high performance lasers, high-efficiency non-linear optic devices and high sensitivity sensors.

The spectral response of the resonator is calculated by filling the surrounding medium with numerous RIs. The normalized intensity at the output port of the sensor is calculated for the wavelength range of $600 \div 1200 \mathrm{~nm}$ at $n=1.0,1.1,1.15,1.2,1.25,1.3,1.35$. The parametric sweep function is used to determine the spectral response of the resonator at a wavelength increment of $1 \mathrm{~nm}$. The transmission spectrum of a standard ring resonator design in the presence of different values of $n$ is plotted in Fig. 2a, which shows a prominent redshift of $\lambda_{\text {res }}$. Moreover, $\lambda_{\text {res }}$ dependence on the NDs embedded cavity and the radius $(r)$ of the NDs are also studied and presented in Fig. 2b, which also demonstrates a redshift as $r$ increases.
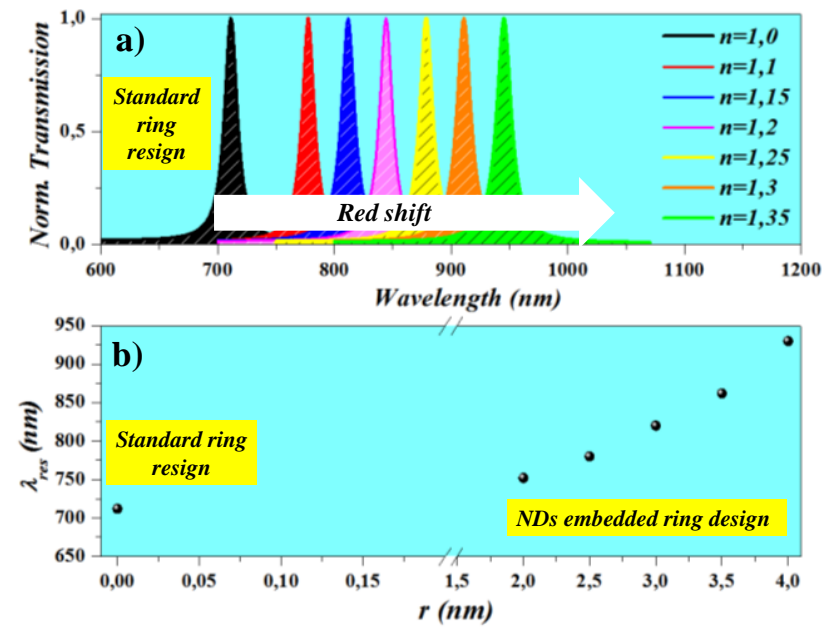

Fig. 2. a) Transmission spectrum of a standard ring resonator for several ambient refractive indices, b) $\lambda_{\text {res }}$ dependent on the radius $(r)$ of NDs embedded cavity.

In Fig. 3a , the sensitivity is calculated for the number of NDs embedded in the cavity. In the cavity, the first seven NDs are embedded in one side length of the ring and then added in the other three sides periodically. The radius $(r)$ of the NDs is set at $2.5 \mathrm{~nm}$. We can see an obvious enhancement of sensitivity with the incorporation of the NDs in the cavity. The best sensitivity $\left(S_{\max }\right)$ of the sensor obtained in Fig. 3a is presented in Table 2.

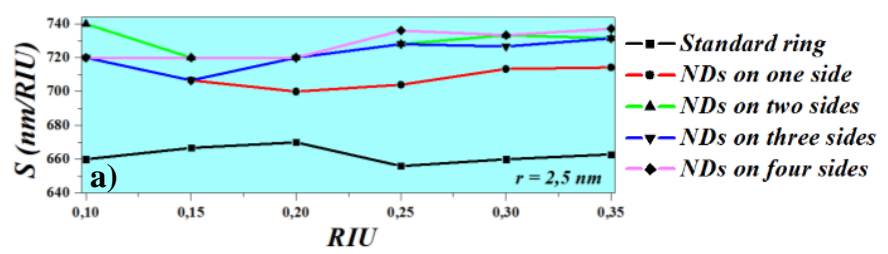




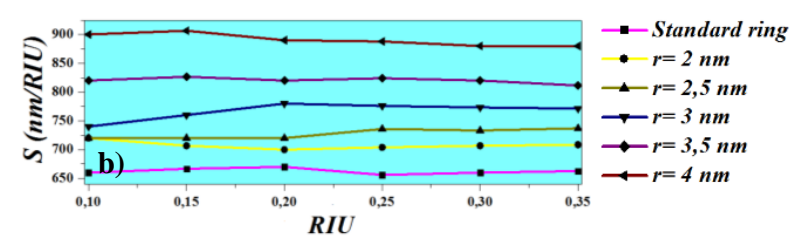

Fig. 3. The sensitivity of the SRR by placing NDs of a) $r=2.5 \mathrm{~nm}$ in different sides of the cavity. b) various sizes in all four sides of the cavity.

Table 2. The sensitivity of the NDs embedded SRR design.

\begin{tabular}{|c|c|c|c|c|c|}
\hline & $\begin{array}{c}\text { Standard } \\
\text { ring }\end{array}$ & $\begin{array}{c}\text { NDs in } \\
\text { one } \\
\text { side }\end{array}$ & $\begin{array}{c}\text { NDs in } \\
\text { two } \\
\text { sides }\end{array}$ & $\begin{array}{c}\text { NDs in } \\
\text { three } \\
\text { sides }\end{array}$ & $\begin{array}{c}\text { NDs in } \\
\text { four } \\
\text { sides }\end{array}$ \\
\hline $\begin{array}{c}S_{\max } \\
(\mathrm{nm} / \mathrm{RIU})\end{array}$ & 670 & 720 & 740 & 732 & 738 \\
\hline
\end{tabular}

The $S, Q$-factor and $F O M$ of the resonator are also related to the size of the NDs. Therefore, we considered the effect of the radius of NDs on spectral performance by maintaining the NDs number at 28 (7 on each side of the ring) as shown in Fig. 3 b. The $S_{\max }, F O M$ and $Q$-factor of the resonator depending on NDs radius $(r)$ are presented in table 3 .

Table 3. Spectral performance of the proposed sensor design

\begin{tabular}{|c|c|c|c|c|c|c|}
\hline Parameters & $\begin{array}{c}\text { Standard } \\
\text { ring }\end{array}$ & $\begin{array}{c}\mathrm{NDs} \\
\mathrm{r}=2 \\
\mathrm{~nm}\end{array}$ & $\begin{array}{c}\mathrm{NDs} \\
\mathrm{r}=2.5 \\
\mathrm{~nm}\end{array}$ & $\begin{array}{c}\mathrm{NDs} \\
\mathrm{r}=3 \\
\mathrm{~nm}\end{array}$ & $\begin{array}{c}\mathrm{NDs} \\
\mathrm{r}=3.5 \\
\mathrm{~nm}\end{array}$ & $\begin{array}{c}\mathrm{NDs} \\
\mathrm{r}=4 \\
\mathrm{~nm}\end{array}$ \\
\hline $\begin{array}{c}\boldsymbol{S}_{\max } \\
(\boldsymbol{n m} / \boldsymbol{R I U})\end{array}$ & 670 & 720 & 738 & 780 & 827 & $\mathbf{9 0 7}$ \\
\hline $\boldsymbol{Q}$-factor & 52.4 & $\mathbf{5 2 . 6}$ & 45.9 & 46.6 & 45.7 & 41 \\
\hline $\boldsymbol{F O M}$ & 49.2 & $\mathbf{5 0 . 4}$ & 43.4 & 44.3 & 43.7 & 40.8 \\
\hline
\end{tabular}

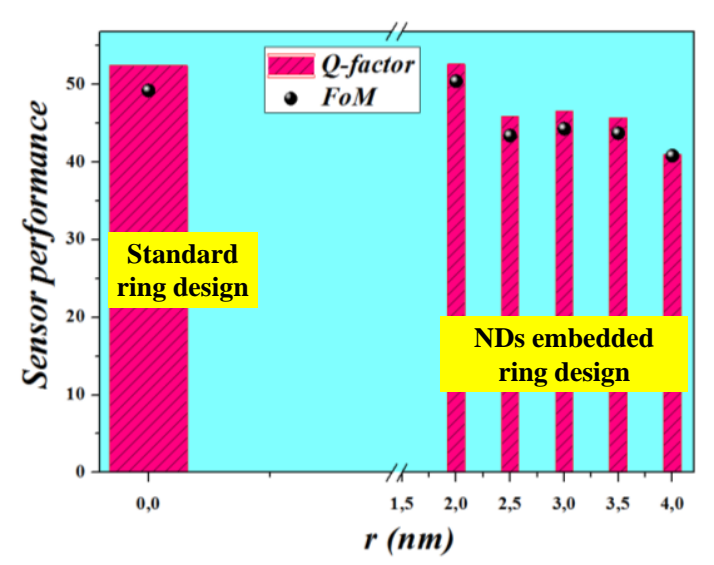

Fig. 4. FoM and $Q$-factor dependence on the radius ( $r$ ) of NDs.

With an increasing size (r) of the NDs, the FOM and $Q$ factor of the resonator decrease while a significant enhancement in $S$ is observed. The decrease in the FOM and $Q$-factor is related to the broadening of the FWHM. Therefore, there is a compromise between $S$ and FoM and $Q$-factor. The maximum $F O M$ and $Q$-factor of 50.4 and 52.6 are obtained for the resonator having NDs of $r=$ $2 \mathrm{~nm}$ as shown in Fig. 4, whereas $S_{\max }=907 \mathrm{~nm}$ is attained for the NDs of $r=4 \mathrm{~nm}$ due to the intensification of SPPs at the boundary of the MIM bus WG. These spectral characteristics are higher than those reported in several recent works based on MIM structures [7, 14-16].

In conclusion, we presented a highly sensitive and compact sensor design based on Nano-dots incorporated in a metal-insulator-metal square ring resonator. The sensitivity is boosted due to the array of Nano-dots periodically placed in the cavity which forms a grating and intensifies the SPPs at the boundaries of the dots. The sensitivity of the device can be enhanced by increasing the number and size of the Nano-dots. The maximum sensitivity of $907 \mathrm{~nm} / \mathrm{RIU}$ is obtained by incorporating 28 Nano-dots of a $4 \mathrm{~nm}$ radius in the standard rind resonator. These results are beneficial for a compact and high sensitive sensor and may advance the integration level of integrated optical circuits.

This work was financially supported by the Ministry of Science and Higher Education within the State assignment FSRC "Crystallography and Photonics" RAS (No. 007-GZ/Ch3363/26).

\section{References}

[1] Z. Han, S.I. Bozhevolnyi. Rep. Prog. Phys. 76, 016402 (2013).

[2] N.L. Kazanskiy, S.N. Khonina, M.A. Butt, Physica E 117, 113798 (2020).

[3] D.K. Gramotnev, S.I. Bozhevolnyi. Nat. Photonics 4, 83 (2010).

[4] A.N. Taheri, H. Kaatuzian. Appl. Opt. 53, 28 (2014).

[5] P. Neutens, L. Lagae, G. Borghs, P.V. Dorpe. Opt. Expr. 20, 4 (2012).

[6] M.A. Butt, S.N. Khonina, N.L. Kazanskiy. Waves in Random and complex media, doi. org/10.1080/17455030.2019.1568609

[7] M.A. Butt, S.N. Khonina, N.L. Kazanskiy. J. Modern Opt. 65, 1135 (2018).

[8] M.A. Butt, S.N. Khonina, N.L. Kazanskiy, Highly sensitive refractive index sensor based on hybrid plasmonic waveguide microring resonator, doi. org/10.1080/17455030.2018.1506191

[9] Y. Fang, M. Sun, Light: Sciene Appl. 4, e294 (2015).

[10] H. Lu, G.X. Wang, X.M. Liu. Chin. Sci Bull., doi: 10.1007/s11434013-5989-6

[11] J.N. Anker et al., Nature Mat. 7, 442 (2008).

[12] M.A. Butt, S.N. Khonina, N.L. Kazanskiy. J. Modern Opt. 66, 1038 (2019).

[13] Z.-D. Zhang, H.-Y. Wang, Z.-Y. Zhang. Plasmonics 8,797 (2013).

[14] Y. Yu, J. Si, Y. Ning, M. Sun, X. Deng. Opt. Lett. 42, 187 (2017).

[15] B.H. Zhang, L-L. Wang, H-J. Li et al., J. Opt. 18, 065001 (2016).

[16] X. Zhao, Z. Zhang, S. Yan, Sensors 17, 1494 (2017).

[17] J. Zhou et al., AIP Advances 7, 015020 (2017).

[18] V. Perumal, U. Hashim. J. Appl. Biomed. 12, 1 (2014).

[19] H.Gai, J. Wang, Q. Tian, Appl. Opt. 46(12), 2229 (2007). 